\title{
WiSlow: A WiFi Network Performance Troubleshooting Tool for End Users
}

\author{
Kyung-Hwa Kim \\ Columbia University \\ New York, NY, USA \\ Email:khkim@cs.columbia.edu
}

\author{
Hyunwoo Nam \\ Columbia University \\ New York, NY, USA \\ Email: hn2203@columbia.edu
}

\author{
Henning Schulzrinne \\ Columbia University \\ New York, NY, USA \\ Email: hgs@cs.columbia.edu
}

\begin{abstract}
The increasing number of 802.11 APs and wireless devices results in more contention, which causes unsatisfactory WiFi network performance. In addition, non-WiFi devices sharing the same spectrum with 802.11 networks such as microwave ovens, cordless phones, and baby monitors severely interfere with WiFi networks. Although the problem sources can be easily removed in many cases, it is difficult for end users to identify the root cause.
\end{abstract}

We introduce WiSlow, a software tool that diagnoses the root causes of poor WiFi performance with user-level network probes and leverages peer collaboration to identify the location of the causes. We elaborate on two main methods: packet loss analysis and 802.11 ACK pattern analysis.

\section{INTRODUCTION}

Today, it is common for households to put together home wireless networks with a private wireless router (access point) that supports multiple wireless devices. However, the increasing usage of wireless networks inevitably results in more contention and interference, which causes unsatisfactory WiFi performance. There are two main sources of performance degradation. First, WiFi devices connected to the same AP or nearby APs that use the same channel can cause packet collisions, i.e., channel contention. Second, non-WiFi devices such as microwave ovens, cordless phones, and baby monitors emit severe interference because these devices operate on the same $2.4 \mathrm{GHz}$ spectrum as $802.11 \mathrm{~b} / \mathrm{g}$ [6]. Although these problem sources can be easily removed in many cases (e.g., by relocating the baby monitor, choosing a different channel, or moving to the $5 \mathrm{GHz}$ band), it is difficult for technically nonsavvy users to even notice the existence of channel contention or non-WiFi interference. Instead, properly working routers or service providers are frequently misidentified as the culprit while the actual root cause remains unidentified. However, isolating the root causes of poor WiFi performance is nontrivial, even for a network expert, because they show very similar symptoms at the user level, and special devices are required in order to investigate the lower layers of the protocol stack.

We introduce WiSlow ("Why is my WiFi slow?"), a software tool that diagnoses the root causes of poor WiFi performance with user-level network probes and leverages peer collaboration to identify their location. We focus on building software that does not require any additional spectrum analysis hardware (unlike, e.g., WiSpy [4], AirSleuth [2], or AirMaestro [1]). In addition, WiSlow does not depend on a specific network adapter such as the Atheros chipset, which were used to achieve similar goals in other studies [10], [11]. WiSlow runs on a typical end user's machine. Currently, it runs on any machine that supports wireless packet sniffing enabled by the 802.11 monitor mode. We trace behaviors of 802.11 networks such as retries, Frame Checksum Sequence (FCS) errors, packet loss, and bit rate adaption, which can be observed on ordinary operating systems. Our experimental results show that the statistical patterns of the above variables vary depending on the problem sources. For example, in the case of non-WiFi interference, we observed a greater number of retried packets, fewer FCS errors, and larger variations in the bit rates compared to channel contention. Correlating these variables, we can categorize the sources of performance problems into several distinct groups. In addition, the nonWiFi devices such as baby monitors, cordless phones, and microwave ovens have different patterns when the number of UDP packets and 802.11 ACKs are plotted over time. In this study, we elaborate on two main methods: packet loss analysis and 802.11 ACK pattern analysis. To improve the accuracy of the algorithm, WiSlow also uses a heuristic method that considers the history of interference episodes and matches it to the common usage characteristics of various devices (e.g., microwave ovens are often used intermittently for periods of 5-30 minutes, whereas baby monitors are used continuously) to ascertain the source of the problem.

Based on our experimental results and heuristic methods, we have developed an algorithm that successfully distinguishes channel contention from non-WiFi interference and infers the product type of the offending device. We believe that this technology will be useful to end users since it can inform them of what needs to be done in order to improve the performance of their networks-whether to upgrade their Internet bandwidth or remove a device that is emitting the interference.

In non-WiFi interference scenarios, another goal is to identify the location of the source of interference. Although it is difficult to pinpoint the exact physical location of the source without the support of hardware or APs, we can infer the relative location of the problem source by collaborating with other end users connected to the same wireless network. WiSlow collects patterns of variables from peers and determines whether others observe the interference at the same time. If all the machines observe it, it is highly likely that the problematic source is close to the wireless AP. However, if only one of the peers observes the interference, the source is likely to be located close to that peer. Our experimental results clearly show that this approach is feasible.

In summary, WiSlow uses information obtained from captured packets and other users to (i) distinguish channel contention from non-WiFi interference, (ii) infer the product 
type of the interfering device (e.g., a microwave oven, cordless phone, or baby monitor), and (iii) point out the approximate location of the source of interference. We developed and evaluated an implementation of WiSlow that diagnoses the cause of WiFi performance degradation and returns reports to users such as "It appears that a baby monitor located close to your router is interfering with your WiFi network."

The remainder of this paper is structured as follows. In Section II, we describe the common sources of WiFi performance degradation. In Section III, we discuss the restrictions of an end user's environment and how WiSlow attempts to overcome them. Section IV explains the detailed methods of WiSlow. Finally, Section VII summarizes our conclusions.

\section{BACKGROUND}

Common sources of $\mathrm{WiFi}$ performance degradation include:

- WiFi channel contention: degradation due to a channel crowded by multiple WiFi devices that compete to transmit data through an AP. In addition, interference due to nearby APs that are using the same channel or adjacent channels cause the similar performance problems since the APs share the limited capacity of the channel. We use the term contention in this paper to refer to this type of performance degradation.

- Non-WiFi interference: interference due to non-WiFi devices that use the same $2.4 \mathrm{GHz}$ spectrum as the $802.11 \mathrm{~b} / \mathrm{g}$ networks such as microwave ovens, cordless phones, baby monitors, and Bluetooth devices. In this paper, we use the term interference to refer to this type of degradation.

- Weak signal: when the signal is not strong enough due to distance or obstacles, packets can be lost or corrupted.

Although the extent varies, all the above sources result in severe performance degradation-some of them even drop the TCP/UDP throughput to almost zero [10]. In this study, we focus on WiFi channel contention and common non-WiFi interference sources.

\section{Challenges}

In this section, we describe several difficulties analyzing wireless networks due to end users' restricted conditions such as limited hardware capabilities and lack of monitoring data.

\section{A. Inaccurate RSSI and SINR measurement}

Received Signal Strength Indication (RSSI) and Signal-toInterference-plus-Noise Ratio (SINR) are generally considered to be the key factors that indicate the quality of a wireless link. However, according to Vlavianos et al. [12], RSSI inaccurately captures the link quality and it is difficult to accurately compute SINR with commodity wireless cards, thus they are not appropriate when estimating the quality of the link. We also observed a similar result when monitoring RSSI and SINR values. We placed various types of interference sources close to the AP and measured the values on a general client machine ${ }^{1}$. In Figure 1a, RSSI values with a baby monitor were consistently higher than a cordless phone, which should be

\footnotetext{
${ }^{1}$ We used a MacBook Pro 2013 (network card: AirPort Extreme, chipset: Broadcom BCM43 series) in this measurement
}

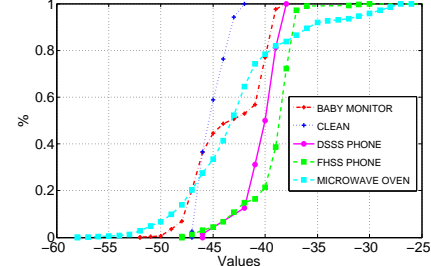

(a) RSSI measurement

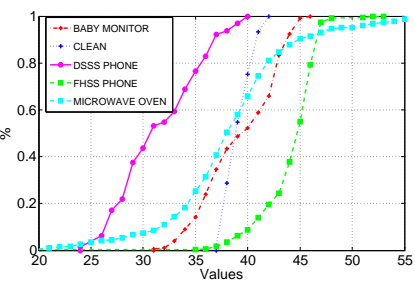

(b) SINR measurement
Fig. 1: Cumulative distribution functions of RSSI and SINR values

reversed when the measured UDP throughput is considered. In, Figure $1 \mathrm{~b}$, the SINR values with a cordless phone were higher than even a no-interference case. Furthermore, these results varied for each experiment. Based on this observation, we conclude that RSSI and SINR values captured by a general wireless cards do not correctly represent the level of interference. Therefore, we do not use these metrics for other purposes besides as a hint in the case of an extremely weak signal.

\section{B. No specific network adapter or driver}

We do not make any assumptions about the specific network adapters or drivers that end users may have. Some Atheros chipsets, which are widely used in research studies, support a spectral scan that provides a spectrum analysis of multiple frequency ranges. Rayanchu et al. developed Airshark [10] and WiFiNet [11] leveraging this feature to distinguish non-WiFi interferers using a commodity network card without specialized hardware. Since our tool aims to provide the best estimation of the problem source, if the user happens to have this specific network adapter, WiSlow can adopt the same approach. However, to the best of our knowledge, only a few chipsets currently provide this feature. In addition, we failed to discover references to this feature for any OS other than Linux. Since there are hundreds of products that use a different chipset and/or OS, it is impractical to assume that a general end user has this specific setup. Therefore, we focus instead on analyzing the quality of a link using user-controllable protocols such as UDP and 802.11 packets. Because the mechanisms of these protocols are not significantly different for many WiFi devices, we believe WiSlow can help a wider range of end users.

\section{Difficulties in obtaining multiple channel information}

Without special hardware or a particular network adapter, it is still possible to measure signal strength by monitoring 802.11 packets. In addition, signal information from multiple frequency bands can be obtained by channel switching. It may help to identify the signal signature of each interfering device. However, without the specialized functionalities of some wireless cards, the AP must be reset whenever the channels are switched. This is not practical for general client machines, not only because it takes a while to scan all the channels, but also because the frequencies given for the signal samples are not at a sufficiently fine-grained resolution. Therefore, we assume that we can only observe a fixed channel of the network. The disadvantage of this approach is that it may fail to detect some interferers that operate within another range of 
frequencies. However, it is reasonable for WiSlow to ignore this case because there is no motivation from an end user's perspective to detect these interferers if the interference does not overlap with his/her WiFi network.

\section{Lack of monitoring data}

Another restriction in the end-user environment is the lack of a monitoring history. If we assume that we have been monitoring the machine up to the moment when a performance problem happens, the diagnosis will be easier because we can obtain several important clues such as the average quality of the network's usual performance, the time when the problem started, and whether it has happened in the recent past. However, although the overhead of network monitoring is not heavy on modern machines, it is difficult to expect that end users will continuously run such a tool. The more common scenario is that a user launches a troubleshooting tool like WiSlow and requests a diagnostic only after he/she has noticed a severe performance problem. Therefore, we need to design the tool assuming little or no previous monitoring data. In the next section, it is explained how WiSlow estimates the problem source without knowing the baseline quality of the network.

\section{WISLOW}

In this section, we elaborate on the details of probing methods for identifying the root causes of network interference. First, to investigate the behavior of WiFi networks in each problem scenario, we artificially inject problems while transmitting UDP packets between a client (laptop) and an AP, capturing every packet on the client. Then, we trace the transport layer (UDP), the 802.11 MAC layer, and some useraccessible 802.11 PHY layer information to ascertain each problematic scenario's interference levels and characteristics.

To capture 802.11 packets, WiSlow leverages the monitor mode that provides the Radiotap [3] header, a standard for 802.11 frame information. The headers are used to extract the lower layer information such as FCS errors and bit rates. Sniffing the wireless packets is supported by most Linux and all Mac OS X machines without additional driver or kernel modification. Therefore, if we can successfully characterize each performance-degrading source by probing the transmitted packets, the same probes will enable WiSlow to identify the problem on most platforms. However, it is not always possible to capture wireless packets on some types of OS, e.g., Microsoft Windows [5]. Instead, Windows provides several APIs that report 802.11 packet statistics to user applications. Those APIs enable WiSlow to run on Windows because they provide all the information that WiSlow must extract from the 802.11 packets. In summary, WiSlow can operate properly if the client's machine supports wireless packet sniffing or provides a set of appropriate APIs.

In the following sections, we explain WiSlow's two main diagnostic methods, packet loss analysis and ACK pattern analysis.

\section{A. Packet loss analysis}

First, we found that each problem source varies in their packet loss characteristics, represented by three statistics: 1) the number of 802.11 retries, 2) the number of FCS errors, and 3 ) the bit rates. In each experiment, we measured these values on a client laptop while downloading $100 \mathrm{MB}$ of packets from an AP. The values were recorded for each $100 \mathrm{~KB}$ of UDP packets received. Thus, we collected a total of 1,000 samples for each experiment. We repeated this experiment for different scenarios including channel contention and non-WiFi interferences. To simulate channel contention, we set up several laptops sending bulk UDP packets to the AP. To generate non-WiFi interference, we placed each interfering device (baby monitors, microwave ovens, and cordless phones) close to the AP (about $20 \mathrm{~cm}$ ) and measured the effect on the client placed at various distances from the AP, e.g., $5 \mathrm{~m}, 10 \mathrm{~m}$, and $20 \mathrm{~m}$. (In this study, we did not consider the simultaneous interference of multiple devices.) Note that the client downloaded $100 \mathrm{MB}$ of UDP packets for each experiment to collect a statistically meaningful amount of samples, but when actually probing on an end user's machine, WiSlow only needs to transmit 10 MBytes of packets to identify the root cause, which takes reasonable amount of time (20-50 s) for a problem diagnosis application.

1) Retry and bit rate: Since an 802.11 retry and bit rate reduction are both initiated by a packet loss, their temporal changes are closely correlated; when a packet loss occurs, the bit rate decreases by the 802.11 rate adaption algorithm. The probability of packet loss then decreases due to the reduced bit rate, which lowers the number of retries. After that, the bit rate gradually increases again due to the reduced packet loss, which leads to a higher probability of packet loss and retries. In other words, if contention or interference exists, it causes packet losses, and then the bit rate and number of retried packets repeatedly fluctuate during the subsequent data transmission. The more interference, the more the fluctuations are observed.

2) FCS errors: Another variable that we trace is the number of FCS errors. Intuitively, it can be predicted that nonWiFi interference introduces more FCS errors than channel contention or a no-interference environment because the packet corruptions are likely to occur more frequently when a medium is noisy. However, in our experiment, it turned out that a large number of FCS errors are not necessarily correlated with severe interference. On the contrary, we often observed that fewer FCS errors occur with severe non-WiFi interference (e.g., interference of a baby monitor) than with channel contention or even a no-interference environment (Figure 2a). This paradox can be explained by the low bit rates in the interference case, which implies that a smaller number of bits are transmitted in the same bandwidth. Consequently, the number of FCS errors alone is not enough to characterize interference sources.

3) Packet loss estimation: As we stated above, although the number of retries, bit rate, and FCS errors are affected by the current state of the wireless network, they often show very different statistics for each experiment set. We conjecture several reasons; the environment is not exactly the same in every experiment, the occurrence of packet loss is probabilistic rather than deterministic, and the individual variables fluctuate over time, affecting each other and leading to different statistics for a certain period of time. Therefore, it would be more reasonable to compare the combinations of these statistics together instead of investigating each variable individually and consider the distributions of the samples rather than their temporal changes. First, since the retries occur when the packets are lost as well as when FCS errors happen, we can estimate the amount of actual packet loss by subtracting the number of FCS errors from the number of retries (Equation 1). 


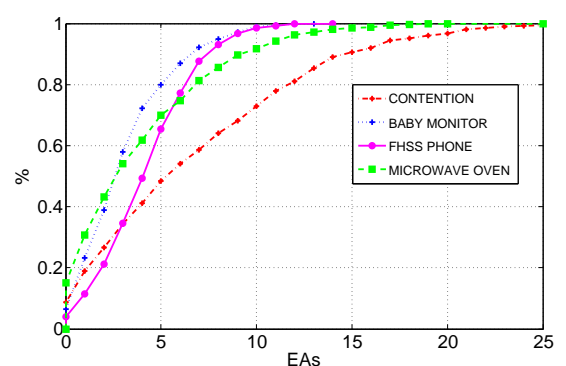

(a) The number of FCS errors per $100 \mathrm{~KB}$

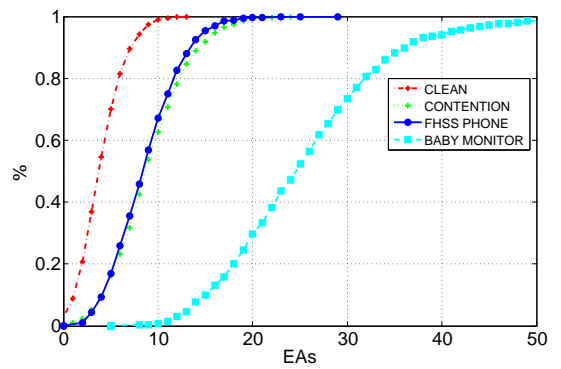

(b) The number of estimated packet loss per $100 \mathrm{~KB}$

Fig. 2: The CDFs of the number of FCS errors and packet loss.

$$
N_{\text {PacketLoss }}=N_{\text {Retries }}-N_{\text {FCSerrors }}
$$

We found that this estimated number of packet losses more reliably represents the level of interference than the individual statistics of retries and FCS errors. In other words, it showed relatively constant results on multiple experiments, while the others varied for each experiment. Figure $2 b$ shows the cumulative distribution function (CDF) of the estimated number of packet loss, where it can be seen that a baby monitor (video transmitter) causes the most severe amount of packet loss while contention and cordless phones cause a relatively small amount of packet loss. Since baby monitors send video and audio data at the same time, they use more bandwidth than cordless phones that send audio only, thus causing more interference. Channel contention has less packet loss because of the 802.11 collision-avoidance functions such as random back-off and RTS/CTS that force each client to occupy the medium in separate time slots. In this case, the divided time slots caused the degradation of throughput, rather than noise from other sources. (The impact of a hidden terminal is not considered in this section.)

Furthermore, we found that the correlation between bit rate and the estimated number of packet losses shows clearer differences among various problem sources. In Figure 3a, the majority of the samples from clean environment are distributed in a healthy zone (higher bit rate and low packet loss) while the samples of baby monitors and microwave ovens are widely dispersed on the coordinate plane. WiSlow uses the correlation of these two variables to distinguish the level of interference.

Although the problem sources each have their own distribution patterns on the above scatter plot, an end user cannot infer a root cause by simply matching the measured statistics with the results of our experiments. This is because

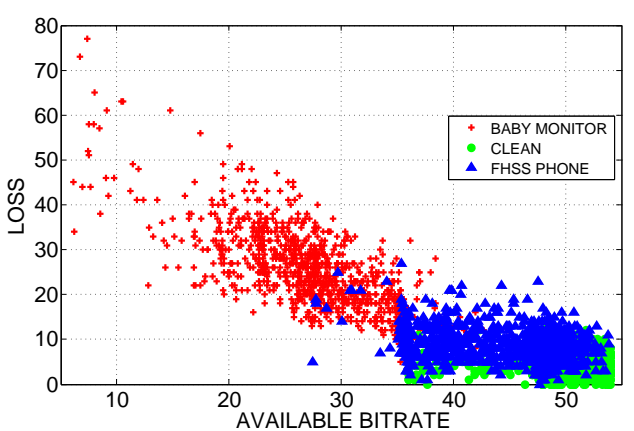

(a) Bit rates and the estimated packet loss with different interference sources

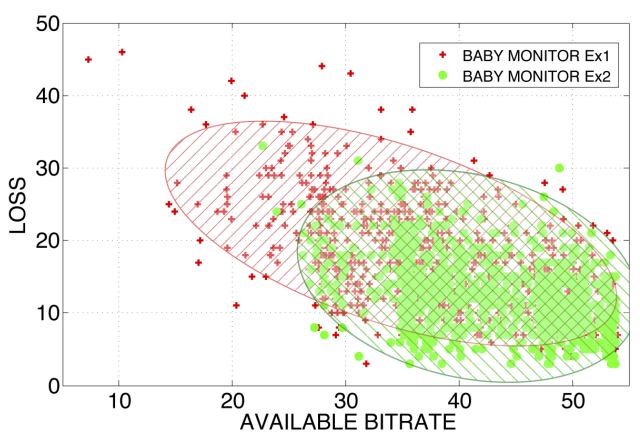

(b) Bit rates and the estimated packet loss with the same device, a baby monitor, in different environment

Fig. 3: The distribution of the correlation of bit rates and the estimated packet loss

the measurement of a wireless network is highly affected by the client's own environment such as distance from the AP, signal power, or fading (multi-path and shadowing). In other words, even though they have the same type of problem, the statistics of the measured metrics can vary depending on each end user's own situation. Note that this is the reason why simple measurements such as the higher-layer throughput (e.g., TCP or UDP) or number of 802.11 retries are not enough to identify the level of interference and the type of interferers. Therefore, to apply our approach to end users, it is important that the measured statistics are independent of the underlying environment. We found that even if the underlying environment changes, the extent of the area, where a set of samples (correlated packet loss and bit rate) are dispersed, remains similar if the problem source is the same. Figure $3 \mathrm{~b}$ shows that even though the two groups of samples from discrete environments are distributed on different spots on the plane, their extent is similar. Thus, we first quantify how widely the samples are dispersed by calculating the Euclidean distances between each sample and the mean. Figure 4 compares the CDFs obtained from two experiments that were conducted with the same baby monitor in two discrete environments. The CDFs of packet loss estimation (Figure 4a) and bit rates (Figure 4b) show different distributions while the CDFs of the Euclidean distances of the samples to the mean show similar distribution (Figure 4c).

Therefore, WiSlow can use the above CDFs of the distances to identify the root causes of network interference. We prepare these CDFs of each problem source in advance, which are obtained from our experiments. Then, WiSlow traces the 


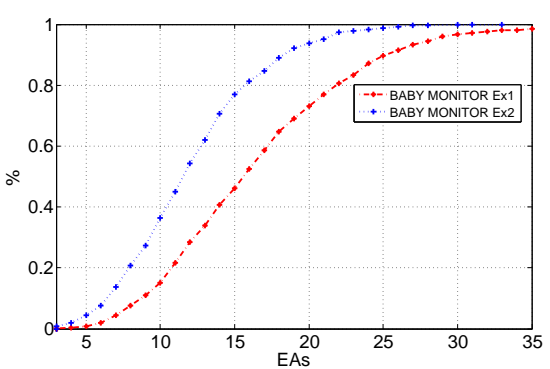

(a) The estimated number of packet loss

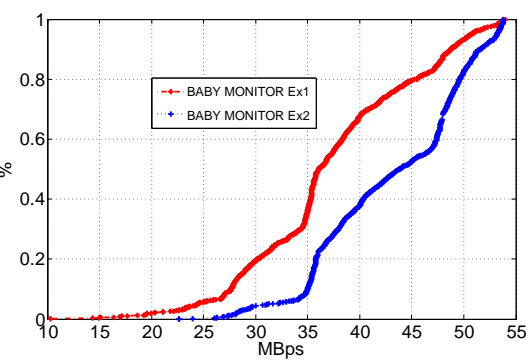

(b) Bit rates

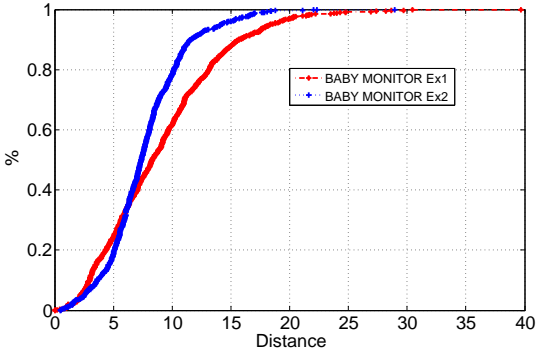

(c) Distance

Fig. 4: The CDFs obtained from two experiments with the same baby monitor in different environment. $100 \mathrm{MB}$ of UDP packets were transmitted from the AP and the values were sampled every $100 \mathrm{~KB}$.

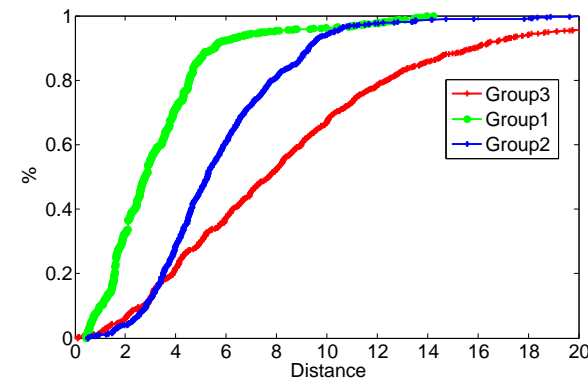

Fig. 5: The packet loss analysis groups the problem sources into three groups: 1) a clean environment, 2) contention and FHSS cordless phones, and 3) microwave ovens and baby monitors

wireless packets on an end user's machine, generates a CDF of the distances, and compares it to the pre-obtained CDFs of each problem source. For the convenience of identification, we group the problem sources into three groups by the shape of the CDFs: no interferers (group 1), light interferers (group 2), and heavy interferers (group 3). Each group has its representative CDFs that are determined by multiple experiments (Figure 5). In our data sets, group 1 indicates a clean environment, group 2 includes channel contention and FHSS cordless phones, and group 3 contains microwave ovens and baby monitors. WiSlow examines which representative $\mathrm{CDF}$ is the most similar one to the user's CDF. To compare the CDFs, WiSlow uses the Two-Sample KolmogorovSmirnov test (K-S test), a widely used statistical method that tests whether the two empirical CDFs obtained from separate experiments have the same distribution [9]. If the p-value of this test is close to 1 , the two CDFs are likely to come from the same distribution, however, if the p-value is close to 0 , they are likely to come from different distributions. Since the K-S test not only considers the average and variance of the samples but also takes into account the shape of the CDFs, it best fits the purpose of WiSlow where it is used to pick the most similar distribution from multiple data sets.

\section{B. ACK Pattern Analysis}

The first method is able to determine which type of loss pattern a problem source has. However, because multiple problem sources are categorized into each group, we need another method that further narrows down the root causes. In this section, we explain the second method, designed to distinguish several detailed characteristics of non-WiFi devices such as frequency hopping and duty cycle.

1) Probing method: WiSlow sends bulk UDP packets to the AP and counts the received 802.11 ACKs to check the quality of a wireless link within a given period. In order to detect patterns on the scale of milliseconds, we use a very small size of UDP packets (12 bytes) that reduces potential delays such as propagation and processing delays, and we transmit as many UDP packets as possible to reduce the intervals between samples. As a result, we received 0-7 ACKs per millisecond with an average number of 2.7 in a clean environment.

In the following sections, we describe the results of the above method when performed with various non-WiFi interferers, and we explain how WiSlow identifies the devices based on the results.

2) Duty cycle (microwave ovens): Microwave ovens generate severe interference in almost every channel of the $2.4 \mathrm{GHz}$ band. We identify this heavy interferer using its duty cycle, which is the ratio of the active duration to the pulse period. It is known that the duty cycle of microwave ovens is fixed at 0.5 and the dwell time is $16.6 \mathrm{~ms}(60 \mathrm{~Hz})^{2}$ [7]. This implies that it stays in the ON mode (producing microwaves) for the first $8.3 \mathrm{~ms}$ and the OFF mode for the next $8.3 \mathrm{~ms}$. This feature can be observed by various means such as using a spectrum analyzer [4] or by signal measurement [10].

Our hypothesis was that a user-level probe could also detect this on-off pattern if the network packets were monitored on a millisecond timescale because the packets would be lost only when the interferer was active (on mode). To validate this assumption, we implemented the above method and plotted the number of successfully received 802.11 ACKs per millisecond. As a result, a clearly perceptible waveform with a 0.5 duty cycle is observed in Figure 6a; the number of ACKs is over five for the first $8 \mathrm{~ms}$ and zero during the next $8 \mathrm{~ms}$. This pattern repeats while the microwave oven is running. This result becomes clearer when it is converted to the frequency domain (Figure 6b). The highest peak is at $60 \mathrm{~Hz}$, which means the exact cycle is $16.6 \mathrm{~ms}$. This number is exactly the same as the known duty cycle of microwave ovens.

Consequently, if a perceptible cycle is detected from this probing method and the period matches a well-known value, WiSlow determines that the current interference is due to a

${ }^{2}$ This frequency could be $50 \mathrm{~Hz}$ in other countries (e.g., Europe and most of Asia) where $50 \mathrm{~Hz} \mathrm{AC}$ power is used. 


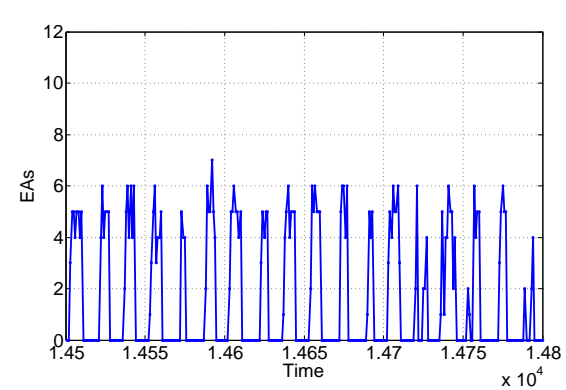

(a) Time domain (Microwave oven)

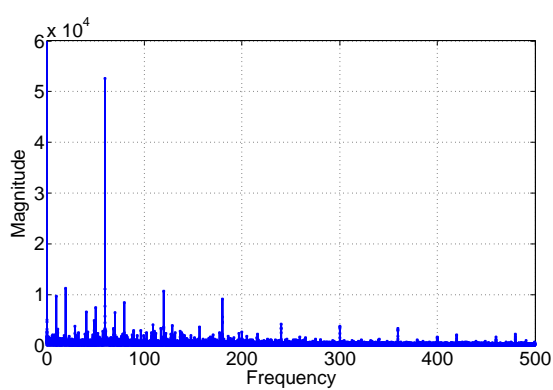

(b) Frequency domain (Microwave oven)

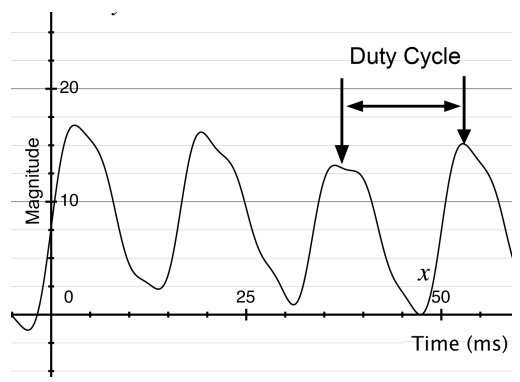

(c) Time domain (Microwave oven) - top 10 frequencies

Fig. 6: The number of 802.11 ACKs per $100 \mathrm{~KB}$ of UDP packets with interference of a microwave oven

particular type of device (e.g., $60 \mathrm{~Hz}$ for microwave ovens).

3) Frequency hopping (baby monitors): The duty cycle of typical video transmitters such as wireless camera is known as one [10]. It means that they send and receive data constantly, implying that they continuously interfere with WiFi networks without any off period. Baby monitors, which transmit video and audio data constantly, also have a similar characteristic. Therefore, intuitively, we do not expect to observe similar ACK patterns as those observed in the microwave oven experiment. However, when converting the plot from the time domain to the frequency domain, we observe another notable pattern. Figure 7a shows that there are multiple high peaks set apart by a specific interval, i.e., $43 \mathrm{~Hz}$ (occurring at 43, 86, 129, and 172 $\mathrm{Hz}$ ). This is in contrast to the microwave ovens that showed only one significantly high peak at $60 \mathrm{~Hz}$ (Figure 6b). We conjecture that these peaks are caused by frequency hopping; a frequency hopper switches its frequency periodically, and interference occurs when it hops to a nearby frequency of the current WiFi channel. However, the frequency-hopping device does not necessarily return to the same frequency at a regular period because the frequency of the next hop is randomly chosen. This randomness instead creates diverse cycles with different periods. However, these periods are multiples of a specific number due to the fixed hopping interval. For clarity, we plot a quantized time-domain graph (Figure 7b) that is converted back from the frequency-domain graph. We used the 10 highest frequencies from Figure 7a. In the time-domain graph, the number of ACKs (y-axis) fluctuates periodically, however, note that the heights of the peaks vary. The possible explanation is as follows: the number of ACKs is large when the device hops far from the current WiFi channel and is relatively small when it hops to a nearby frequency. If the device hops into the exact range of the WiFi channel, the number of 802.11 ACKs drops almost to zero. In other words, there are multiple levels of interference, which depend on how closely in frequency the device hops to the frequency used by the WiFi channel. These multiple levels of interference create several pulses that have different magnitudes and frequencies. Finally, because the hopping interval is fixed, the frequencies of the created pulses are synchronized such that the periods of the cycles are multiples of a specific value.

Consequently, we can distinguish frequency-hopping devices by determining whether the number of 802.11 ACKs has multiple high peaks with a certain interval in the frequency domain. We check this by linear regression of the peak frequencies; if the correlation coefficient is greater than 0.9,
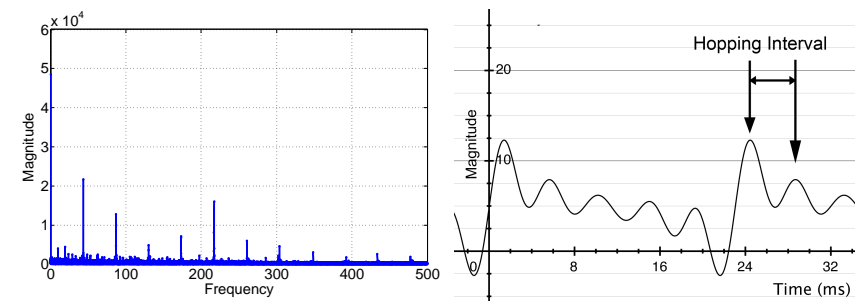

(a) A baby monitor: frequency (b) A baby monitor: time domain domain - top 10 frequencies
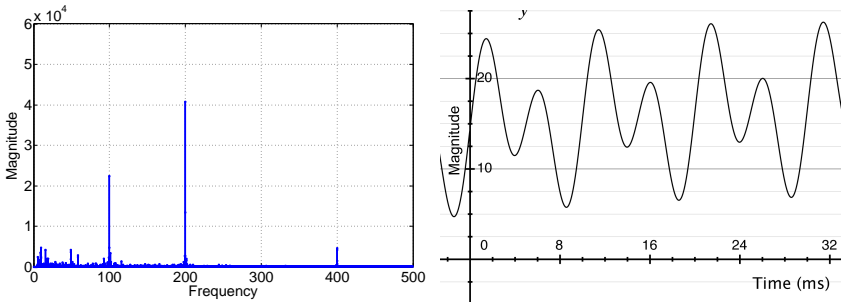

(c) A cordless phone: frequency (d) A cordless phone: time dodomain main - top 10 frequencies

Fig. 7: The number of 802.11 ACKs per $100 \mathrm{~KB}$ of UDP packets with a baby monitor and a cordless phone

we consider it to be a frequency-hopping device.

However, it is obvious that we cannot conclude that every frequency-hopping device is a specific type of device such as a baby monitor. Therefore, WiSlow needs to take into account the results of both the first method and this second method to identify the problem source precisely. For example, if a problem source is classified as group 3 (by the first method) and a frequency hopper (by the second method), we consider it to be a baby monitor. Of course, it is still possible that another type of device not discussed in this study has the same characteristics as a baby monitor. We discuss this case in Section ??.

4) Fixed frequency (analog cordless phones): Typical analog cordless phones use a fixed frequency, so they usually interfere only with a small number of channels. (The analog phones we tested only interfered with Channel 1.) Because they do not change frequency, severe interference occurs if the current WiFi channel overlaps with the frequency of the phone. In addition, their duty cycle is usually one, which implies that no ACK cycle exists. In our experiments, the 


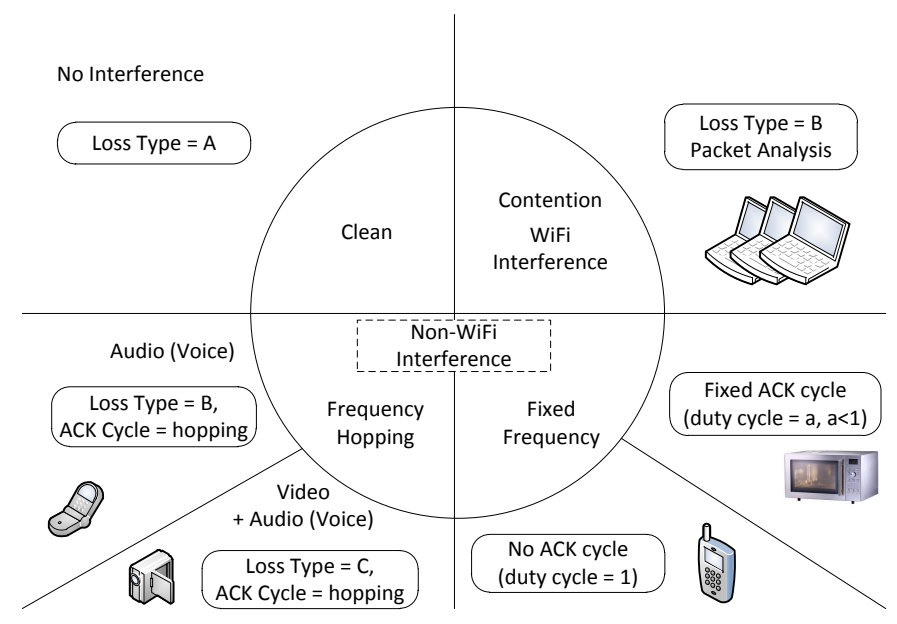

Fig. 8: The classification of problem sources by WiSlow's methods

UDP throughput stayed very low and no explicit ACK cycle (no hopping) was observed, as expected. Therefore, WiSlow determines an analog cordless phone as the interferer if there is severe UDP throughput degradation but no explicit ACK cycle or duty cycle is detected. However, this could be true of other fixed frequency devices such as wireless video cameras that are not discussed in this paper. Currently, when WiSlow detects this type of device, it informs the user that a fixed frequency device has been detected, and suggests that several devices could be the cause, such as analog cordless phones and wireless video cameras.

5) Mixed (hopping and duty cycle): A Frequency Hopping Spread Spectrum (FHSS) phone is another example of a device that explicitly shows the hopping patterns that we described above. In addition, it is known that some FHSS phones have a specific pulse interval, which was verified by Rayanchu et al. [10] using signal measurement. We also confirmed this feature with our user-level probes. Figure $7 \mathrm{c}$ shows the frequency domain of 802.11 ACKs. It shows similar patterns as the microwave ovens (low duty cycle devices) rather than the baby monitors (frequency-hopping devices) even though it also uses frequency hopping. This is because the duty cycle influences the shape of the waveform more than the hopping effect. Therefore, it is possible to use this duty cycle to distinguish the FHSS cordless phones as we did for the microwave ovens. In this case, we use the frequencies, 100 and $200 \mathrm{~Hz}$, to determine the FHSS cordless phone interference. However, to the best of our knowledge, there is no standard regarding the period of the duty cycle for FHSS cordless phones. This means it can vary depending on the product. Therefore, if a duty cycle is detected but the period is an unknown value, WiSlow fails to identify the exact product type. In this case, we provide our best estimate of the problem source by listing a possible set of candidates.

\section{LOCATING INTERFERING DEVICES}

Once WiSlow detects non-WiFi interference and identifies the type of device causing it, the next step is to locate its physical location. Device localization has been investigated intensively in many research fields. A number of research studies on indoor location tracking have tried to pinpoint the location of client laptops or smartphones using various meth-

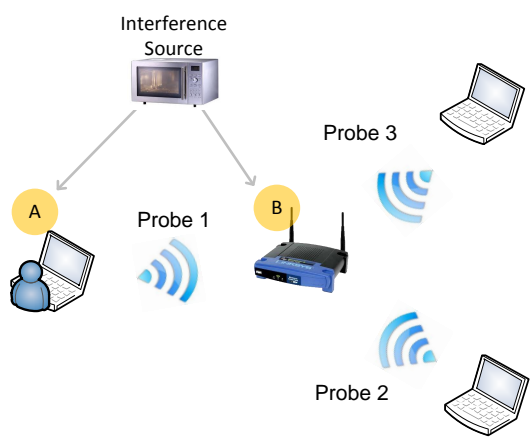

Fig. 9: Probing for localizing an interference source

ods cite. WiFiNet [11] pinpoints the location of the interference source using multiple APs that use Airshark [10]. Although we leverage a similar cooperative diagnosis approach, we focus on the collaboration of multiple end users instead of APs. However, it is not easy to pinpoint the exact physical location of the source by end users because they cannot obtain precise signal information. Instead, we attempt to infer the relative location of the problem source. The basic mechanism is that an end user (probing client) first requests multiple cooperative clients to perform WiSlow diagnostics as described in previous sections. Then it checks whether the other client machines observe the same interference as itself. If all the cooperative client machines observe a particular type of interference at the same time, it is likely that the problematic source is close to the AP because this would affect the entire wireless network. However, if only one of the clients observes the interference, the source is highly likely to be located close to that client.

\section{A. Cooperative Probing}

Figure 9 illustrates the details of the cooperative probing approach. We assume that the clients already have WiSlow installed and have contact information of the others, i.e., IP address and port number. Each probing process takes about 30-40 s; thus, it took a few minutes to collect the results from the three clients in our experiment. Then, WiSlow checks whether the other clients have also detected the same type of interference.

\section{RELATED WORK}

Airshark [10] uses a commodity WiFi network adapter to identify interference sources. It leverages a spectral scan to obtain signal information from multiple frequency ranges. It identifies the interference sources very accurately (over $95 \%$ ) by analyzing the spectrum data using various methods. However, it is not easy to apply this approach for typical end users because collecting high-resolution signal samples across the spectrum is impossible if the network card does not support this functionality.

WiFiNet [11] identifies the impact of non-WiFi interference and finds its location using observations from multiple APs that are running Airshark. Although the authors briefly mention that WiFiNet could be used by end users, they focus more on building infrastructure using APs, while WiSlow focuses on end users and their cooperation to identify the location of the interference source. 
Kanuparthy et al. [8] proposed an approach similar to WiSlow in terms of using user-level information to identify interference sources. They distinguished congestion (channel contention) by measuring the one-way delay of different sizes of packets. Then, they investigated the delay patterns to distinguish a hidden terminal from a weak signal. While this study focused on congestion, weak signals, and hidden terminals, WiSlow focuses on not only congestion and signals but also the detailed identification of non-WiFi interference sources.

\section{CONCLUSION}

We designed WiSlow, a WiFi performance trouble shooting application, specialized to detect non-WiFi interferences. WiSlow distinguishes 802.11 channel contention from non-WiFi interference and identifies the type of interfering devices. We introduced two main methods: packet loss analysis and 802.11 ACK pattern analysis. These methods uses user-accessible packet trace information such as UDP and 802.11 ACKs. In addition, WiSlow leverages peer collaboration to identify the physical location of the sources of WiFi performance degradation.

\section{REFERENCES}

[1] AirMaestro. http://www.bandspeed.com/products/products.php. [Online; accessed May 2013].

[2] AirSleuth. http://nutsaboutnets.com/airsleuth-spectrum-analyzer/. [Online; accessed May 2013].

[3] Radiotap. http://www.radiotap.org/. [Online; accessed May 2013].

[4] Wi-Spy. http://www.metageek.net/. [Online; accessed May 2013].

[5] WLAN packet capture. http://wiki.wireshark.org/CaptureSetup/WLAN. [Online; accessed May 2013].

[6] S. Gollakota, F. Adib, D. Katabi, and S. Seshan. Clearing the RF smog: making 802.11n robust to cross-technology interference. In Proc. of ACM SIGCOMM'11, Toronto, Ontario, Canada, Aug. 2011.

[7] A. Kamerman and N. Erkocevic. Microwave oven interference on wireless lans operating in the $2.4 \mathrm{GHz}$ ISM band. In Proc. of PIMRC '97, Helsinki, Finland, Sep. 1997.

[8] P. Kanuparthy, C. Dovrolis, K. Papagiannaki, S. Seshan, and P. Steenkiste. Can user-level probing detect and diagnose common home-WLAN pathologies. Computer Communication Review, 42(1):715, 2012.

[9] F. J. Massey Jr. The Kolmogorov-Smirnov test for goodness of fit Journal of the American statistical Association, 46(253):68-78, 1951.

[10] S. Rayanchu, A. Patro, and S. Banerjee. Airshark: Detecting non-WiFi RF devices using commodity WiFi hardware. In Proc. of ACM IMC '11, Berlin, Germany, Nov. 2011.

[11] S. Rayanchu, A. Patro, and S. Banerjee. Catching whales and minnows using WiFiNet: Deconstructing non-WiFi interference using WiFi hardware. In Proc. of USENIX NSDI'12, San Jose, CA, USA, Apr. 2012.

[12] A. Vlavianos, L. K. Law, I. Broustis, S. V. Krishnamurthy, and M. Faloutsos. Assessing link quality in IEEE 802.11 wireless networks: Which is the right metric? In Proc. of PIMRC'08, Cannes, France, Sep. 2008 . 